\title{
NEG PUMPING STRIP INSIDE TEVATRON B2 MAGNETS*
}

\author{
Alex Chen, Bruce Hanna, Terry Anderson, FNAL Batavia, IL60510, USA
}

\begin{abstract}
Nonevaporable getter (NEG) pumping strips[1][2][3], 6.2-m long, were installed inside four Tevatron B2 magnets to improve the vacuum environment. This established A linear pumping scheme in the long, unbakable vacuum chamber. The total pressure was reduced significantly.
\end{abstract}

\section{INTRODUCTION}

Some of the highest pressure locations within the Tevatron ring are at the warm B2 magnets located in the E0 warm straight section. The magnets are 20 -feet long with vacuum chambers that can not be easily baked. One SAES' St707 NEG strip, $15 \mathrm{~mm}$ wide and 6.2-m long, was installed in each magnet. The strip extends the entire length of the magnet and provides linear pumping. The St707 NEG strip requires a temperature of $450{ }^{\circ} \mathrm{C}$ for 45 60 minutes for activation, which also provides some degree of internal baking to drive water vapor off the surfaces of vacuum chamber. A DC current of $30 \mathrm{~A}$ was applied for activation.

\section{DESIGN}

A ST707 NEG strip was assembled with a $3 / 4 " \times 1 / 8$ " $\times 246$ " aluminum plate that serves as a strongback. There are standoffs with a ceramic insulator every 4 inches to limit lateral displacement to less than $3 \mathrm{~mm}$ during thermal expansion. Fig. 1 shows the assembly inside the 4" $\times 2$ " (nominal) vacuum chamber. The aluminum strong back rests on the bottom of chamber and is fixed laterally by a stainless steel (SS) wire welded at both ends. The upstream end is fixed by the electric leads to the ultra high vacuum (UHV) feedthrough, and the downstream end is free to move longitudinally for thermal expansion. The assembly occupies about $20 \mathrm{~mm}$ near the outer edge of the $100 \mathrm{~mm}$ lateral aperture. The aperture reduction was equally distributed by re-centering the magnet along the beam centerline.

A small lateral offset of $1.4 \mathrm{~mm}$, between the centerlines of the NEG strip and the aluminum back, was built in to assure the direction of potential movement during cool down is toward the chamber wall, instead of the aperture center.

The assembly has a resistance of $1.0 \Omega$, mainly on the NEG strip substrate, constantan. The NEG strip is connected to DC positive and the aluminum back to DC negative. A DC power supply provides current from 0 30A.

\section{TEST}

A spare magnet was installed with the NEG strip and tested (layout as in Fig. 2) for structural and vacuum characteristics. The structure was subjected to rapid heating (power on at 30A) and cool-down (no ramp down) without causing the assembly to move towards the beam space. It expanded/contracted longitudinally about $3 / 4$ " at the down stream end freely. The strip at full expansion had a lateral displacement of about $2 \mathrm{~mm}$ at the midpoints of the 4" span between spacers, and returned straight after cool-down.

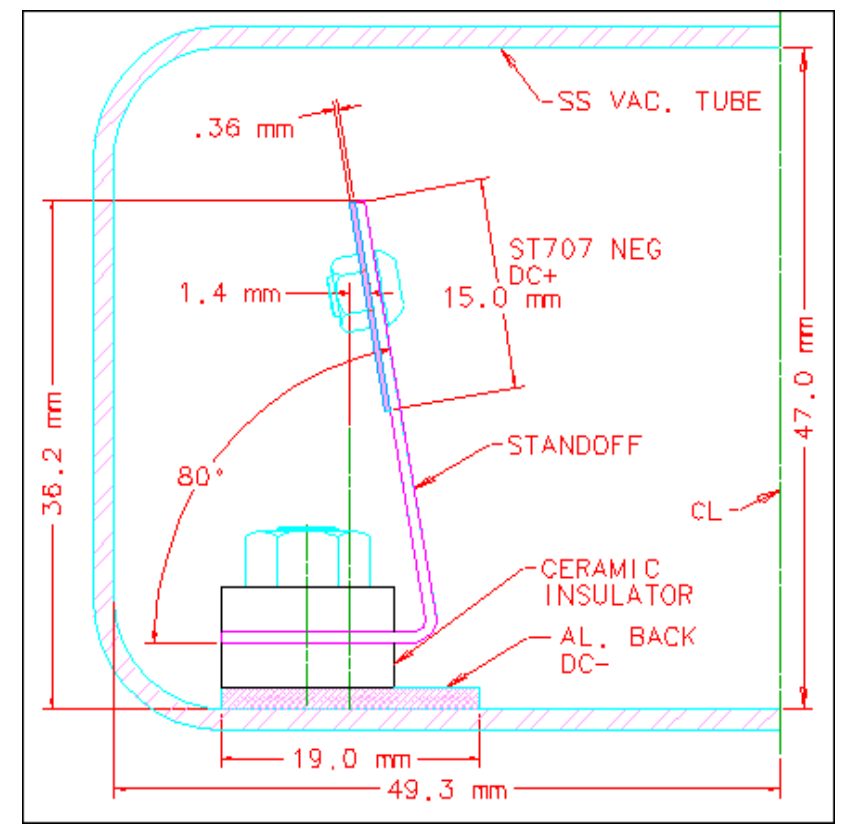

Figure 1: Cross Section View of Half Chamber

The pumping speed and capacity was measured by venting dry nitrogen through a $1 / 8$ " orifice and a variable leak with ion pumps valved out.

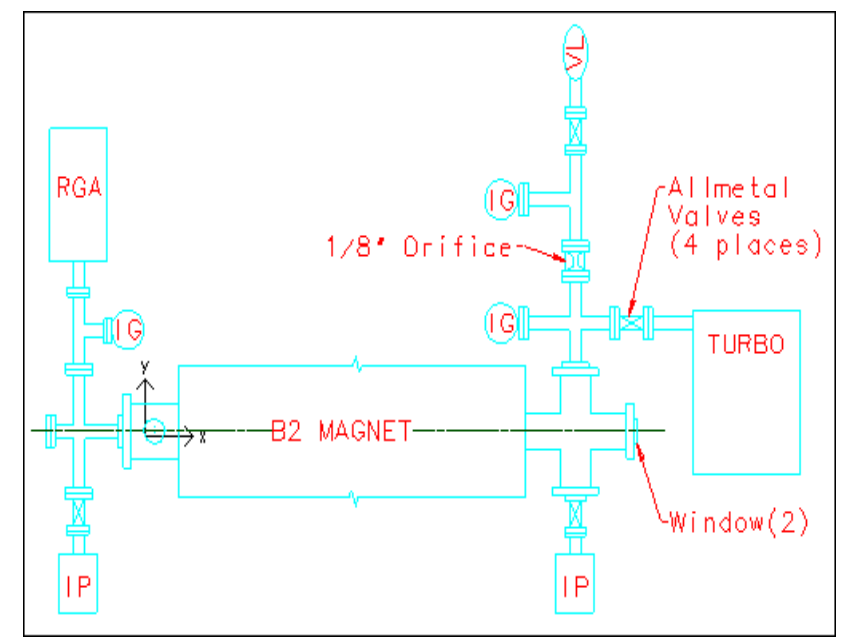

Figure 2: Test Setup 


\section{RESULTS}

The four B2 magnets at Tevatron E0 were installed with NEG strips in November, 2004.

\section{Procedure of Activation}

After installation or venting, it is necessary to activate the NEG strip by heating it to $450{ }^{\circ} \mathrm{C}$ for $45-60$ minutes. A $30 \mathrm{~A}$ DC current is required to heat the strip to this level. In order to eliminate the risk of strip movement toward the beam path during thermal cycles, a procedure was established to control the rate of heating and cooling.

1. Before activating the NEG strip, the vacuum system should be baked. During the system bake, apply 10A to the strip to heat it above $120{ }^{\circ} \mathrm{C}$ (turn off when the system cools down, typically three to five days) to prevent it from adsorbing moisture.

2. During the activation, one or two $20 \mathrm{l} / \mathrm{s}$ ion pumps should be pumping to keep the pressure low, less than 1e-6 Torr.

3. Heating cycle ramp: Increase the current by $5 \mathrm{~A}$ every 5 minutes (or longer if the pressure exceeds 1.0E-6 Torr) until 30A. Stay at 30A for one hour. It may take longer for the first activation after air exposure.

4. Cooling cycle ramp: Decease the current to $20 \mathrm{~A}$ and maintain for 30 minutes, then to $15 \mathrm{~A}$ for 30 minute, $10 \mathrm{~A}$ for 30 minutes, and then to zero.

Because the strip is much thinner than the aluminum back, the strip cools much faster and generates a compressing force on the back. Under this condition the back has the tendency to buckle. Although the buckling is directed towards the wall in this design, this condition needs to be minimized by controlling the cooling rate.

Fig. 3 shows the total pressure profile during the first activation.

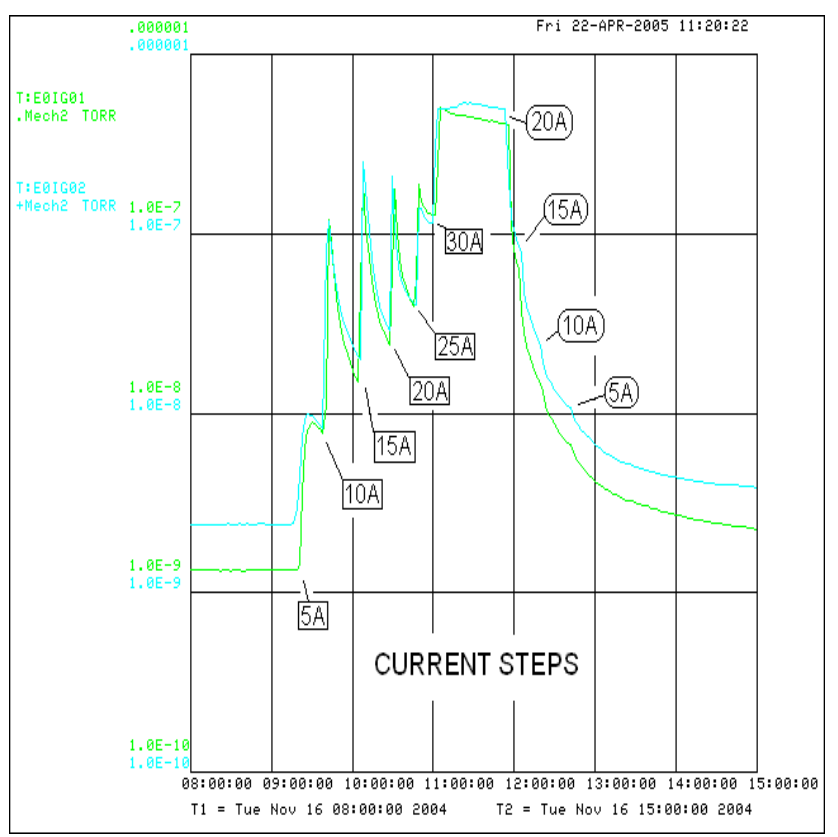

Figure 3: Pressure Changing during Activation

\section{Total Pressure Reduction at E0}

Fig. 4 compares the total pressure reduction between April 2005 and August 2004(before the installation). Total pressure decreased to $7 \mathrm{e}-10$ from $4 \mathrm{e}-9$. The integrated residual gas along the beam path should be reduce even more significantly since with distributed pumping the pressure in the middle of the beam pipe is higher than at the ends[4].

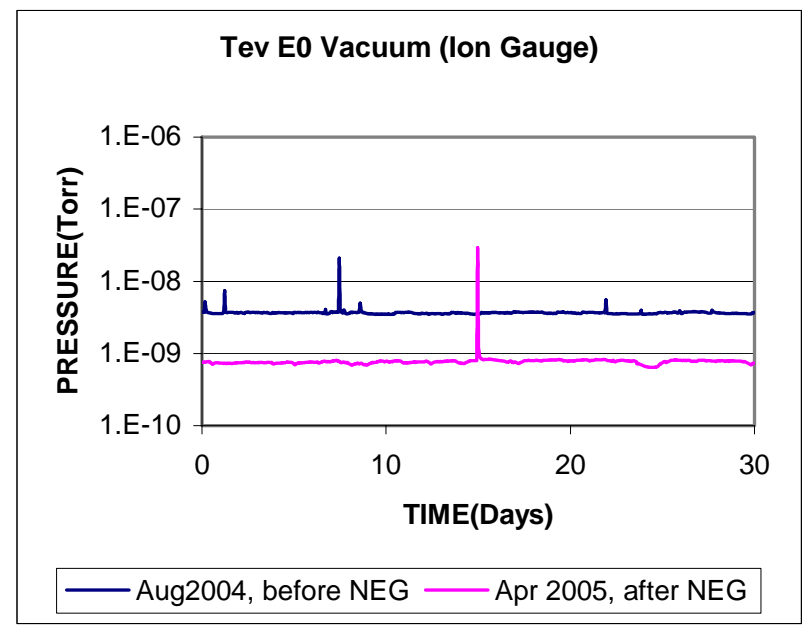

Figure 4: Total Pressure Reduction at Magnets

\section{Pumping Speed \& Capacity}

The strip inside the spare magnet has gone through 12 activation cycles, and its pumping speed has not significantly changed. The initial pumping speed of the $6.2-\mathrm{m}$ strip is above $140 \mathrm{~L} / \mathrm{s}$. Fig.5 shows how the pumping speed changes as a function of the amount of gas (N2) pumped. The flow rate of nitrogen into the system was around 1e-5 Torr-L/s. Based on these results, they will need to be re-generated once a year (during the annual shutdown), and no replacement needed for the projected lifetime of the Tevatron.

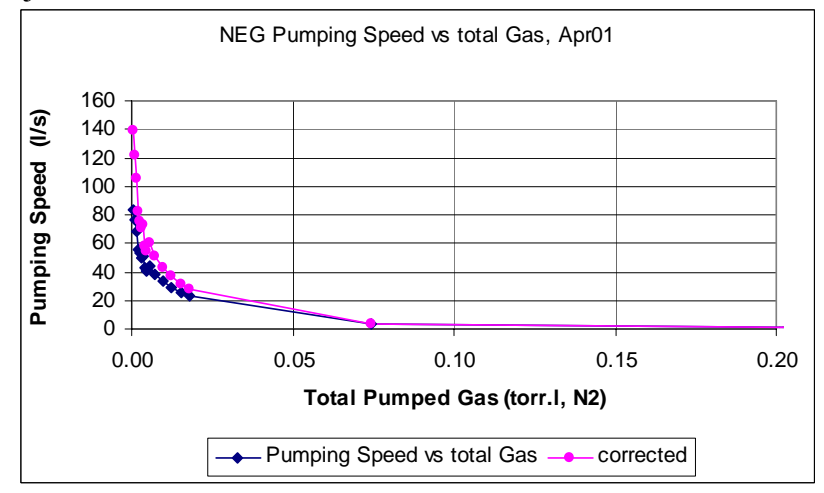

Figure 5: Pumping Speed vs. Gas Deposited

\section{Internal Baking}

Fig.6 shows a RGA scan during the initial activation. The strip has been heated up near $300{ }^{\circ} \mathrm{C}$ with current at 20A. After that, we noted the partial pressure profile of 
water vapor behaved differently from other gas species, it continuously rose without any drop. There was not such rise during repeated activations. Prior to its first activation, it had been about $120^{\circ} \mathrm{C}(10 \mathrm{~A})$ during the system baking. So the continuous rise of water vapor with the current above 20A was not caused by out gassing from the strip. This indicated that the energy radiated from the hot strip, with proper wavelength efficiently absorbed by water vapor[5][6], was high enough to drive water vapor off the chamber surface. So, we believe there was some good internal baking taking place by infrared heating from the hot strip.

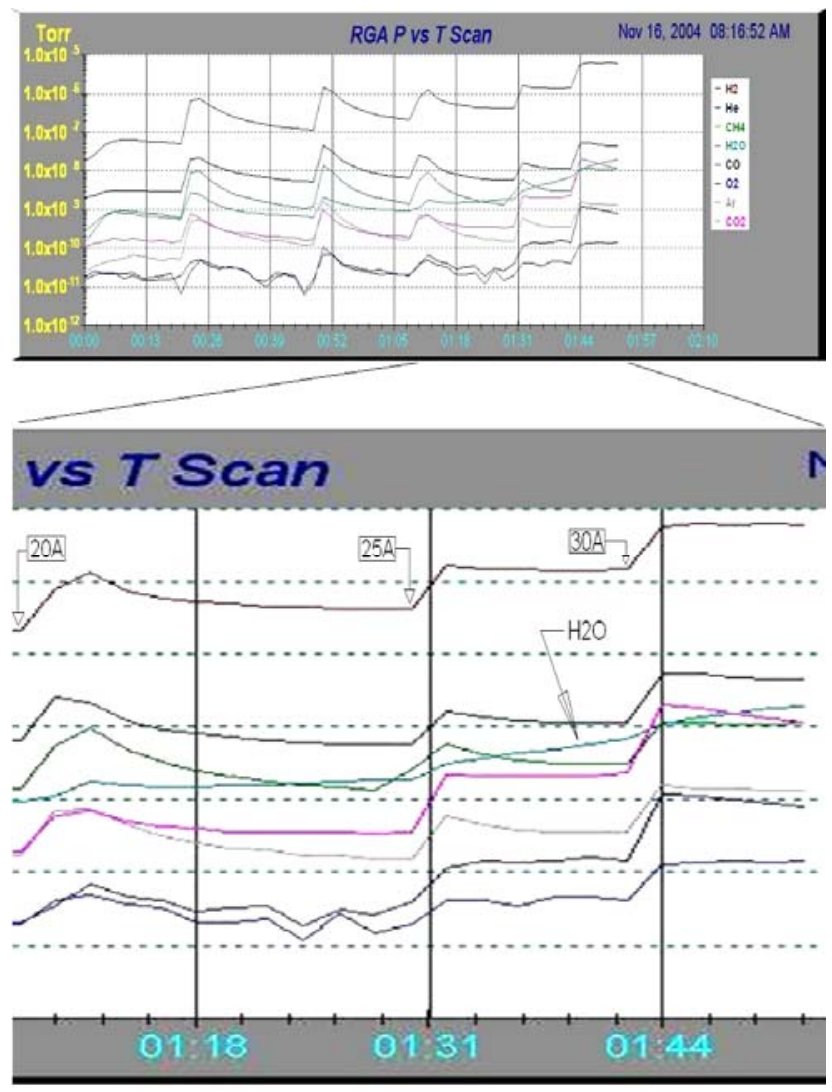

Figure 6: Indication of Internal Baking

\section{CONCLUSIONS}

The vacuum environment at the Tevatron E0 section has been improved by installed NEG strips inside the B2 magnets.

- Total residual gas pressure dropped to $7 \mathrm{e}-10$ from $4 \mathrm{e}-9$ Torr.

- The NEG strip assembly has been reliable structurally.

- The NEG strip provides linear pumping inside the long vacuum chamber.

- Small ion pumps are needed with NEG pumping to pump gas species that NEG does not pump well, such as $\mathrm{He}, \mathrm{Ar}$, and $\mathrm{CH}_{4}$.

- Internal baking shows up above $300{ }^{\circ} \mathrm{C}(20 \mathrm{~A})$.

- Lifetime of ST707 NEG strip is sufficient for Tevatron.

- Proper activation procedure was established.

\section{REFERENCES}

[1] A. Prodromides, "Non-Evaporable Getter Thin Film Coatings for Vacuum Applications" Thesis \# 2652, Lausanne EPFL, 2002.

[2] R. Dortwegt, R. Benaroya, "Distributed Nonevaporable Getter Pumps for the Storage Ring of the APS, P AC1993, p3857.

[3] F. Le Pimpec, "Getters: From Light Bulbs to Accelerators", Fermilab, September 2004.

[4] A. Roth, "Vacuum Technology", $3{ }^{\text {rd }}$ Edition, northholland, 1990.

[5] http//www.lsbu.ac.uk/water/vibrat.html, M. Chaplin, "molecular vibration and absorption of water vapor", 2005.

[6] F. Incropera, D. DeWitt, "Fundamentals of Heat and Mass Transfer", Fifth edition, 2002. 